RECENT ADVANCES

\title{
Biomarkers in paediatric research and practice
}

\author{
B P Lanphear, C F Bearer
}

Arch Dis Child 2005;90:594-600. doi: 10.1136/adc.2003.048819

Children's health is, to a large extent, a function of their environment. Infectious agents remain the leading cause of death and disability in the world. In contrast, many of the new morbidities - asthma, intellectual impairments, behavioural problems, and cancer-are linked with industrial pollutants or other environmental influences. Our understanding of the risk factors for many diseases is incomplete, but it is widely recognised that disability and death result largely from interactions of environmental factors, broadly defined, and host susceptibility. ${ }^{1-3}$

See end of article for authors' affiliations

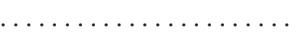

Correspondence to: Dr B P Lanphear, Children's Hospital Medical Center, 3333 Burnet Avenue, Cincinnati, Ohio 45229-3039, USA; bruce.lanphear@chmcc. org

Accepted 23 January 2005
C hildren's environmental health-the study and prevention of disease and illness in children due to unintentional exposure to physical, biological, and chemical agents-is emerging as a new field of research, policy, and clinical practice. ${ }^{4}$ Historically, scientists and physicians have relied on indirect methods to measure environmental influences on children, such as questionnaires, community level monitoring of water and air, or housing condition. ${ }^{5}$ Similarly, we have relied on a child's family history to assess their genetic susceptibility for a particular condition or disease. One of the key innovative tools of this emerging field is the use of biological markers, or biomarkers, to directly measure children's exposure to environmental factors, the effects of exposures, and individual susceptibility, including genetics, to environmental agents $^{6}$ (fig 1).

\section{BACKGROUND}

Infectious agents remain the leading cause of death and disability in the world. In contrast, many of the new morbidities-asthma, intellectual impairments, behavioural problems, and cancer-are linked with non-infectious, environmental agents, or influences. ${ }^{4}$ A century ago, the consequences of children's exposure to toxins, or more correctly toxicants, was poorly understood. In many cases, the effects of environmental toxicants were not detected until they accumulated in the human body in sufficient quantities to cause overt poisoning. Thus, most toxicants were only identified after widespread environmental contamination led to outbreaks of clinical poisonings in children. ${ }^{7}$

Today, it is known that serious health consequences occur not only from heavy exposure, but from low level, chronic exposures to environmental toxicants. Children's exposures to toxicants and the adverse consequences of environmental contaminants are often insidious. Many of these toxicants are widely dispersed in the environment and can readily be found in the blood, body fluids, or tissues of children and pregnant women. ${ }^{4}$ Although the impairments are often subtle for an individual child, the damage can be substantial at the population level, especially when an exposure is prevalent. $^{8}$ Unfortunately, exposures to putative causative agents are often hard to fully characterise because of difficulty in accurately quantifying exposure, estimating the timing of exposure, and measuring the effect of environmental toxicants. ${ }^{9}$

\section{OBSTACLES FOR STUDYING ENVIRONMENTAL TOXICANTS}

Studying the adverse human effects of chronic, low level exposures to environmental toxicants is difficult. There is substantial inter-individual variability in the uptake and effects of toxicants on the fetus and children due to genetic susceptibility, metabolic variation, and nutritional status. ${ }^{6}$ Environmental toxicants tend to cause non-specific deficits or alterations. In contrast with clinical therapeutics, the study of environmental toxicants has relied almost entirely on observational studies and experimental animal models. Finally, it is difficult to distinguish the adverse effects of toxicants from many other social influences because impoverished children are usually more heavily exposed to environmental toxicants. Increasingly, however, researchers are using biological markers to directly measure the actual levels of suspected environmental chemicals in human tissues and fluids, and link these exposures with disability or disease. ${ }^{5} 61011$

\section{BIOMARKERS}

Biomarkers are not new to paediatricians. Paediatricians are quite familiar with using some biomarkers, such as viral serology, to diagnose or treat infections. In contrast, we are less familiar with using biomarkers to identify or quantify children's exposures to environmental influences or industrial pollutants. Biomarkers for environmental toxicants are increasingly being used in paediatric research and practice. Still, despite tremendous promise, there is considerable work needed to make biomarkers of environmental toxicants useful in the clinical setting. Indeed, even though biomarkers are critical to quantify the risk of disease and disability in population based studies, they may not be useful for individual patients. ${ }^{12}$

There are three major types of biomarkers: biomarkers to quantify exposure and internal dose; biomarkers of effect; and biomarkers of susceptibility. ${ }^{6}$ A biomarker of exposure to an exogenous chemical is that chemical, its metabolite(s), or 

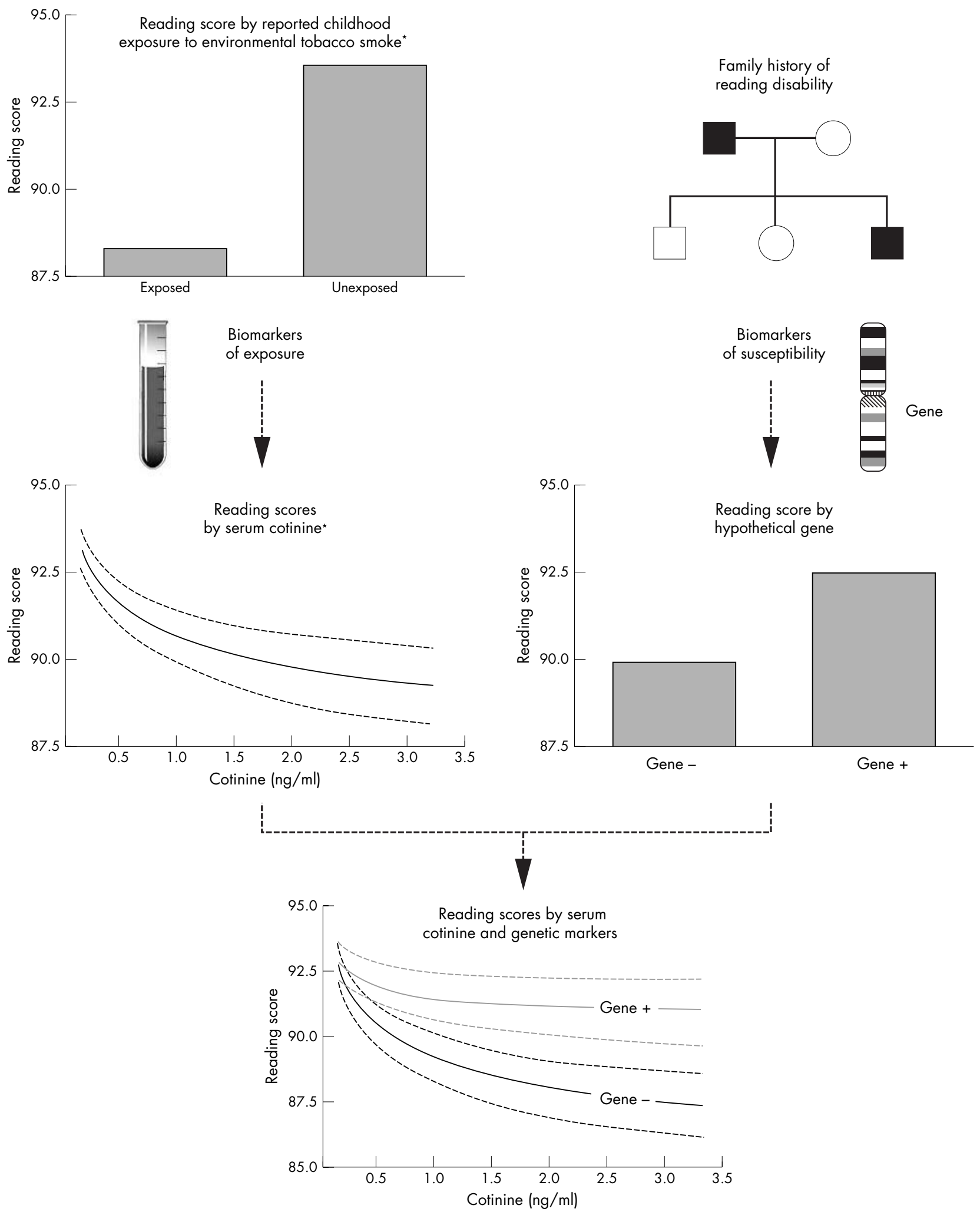

Figure 1 The evolving measures of environmental exposure and genetic susceptibility using biomarkers. *Yolton $\mathrm{K}$ et al. ${ }^{46}$

the product of an interaction between that chemical or metabolite and a target molecule or cell. A biomarker of exposure can be a toxicant (for example, lead or PCBs), a metabolite of the toxicant (for example, cotinine, a metabolite of nicotine), or an early reaction to the toxicant (for example, induction of P450 enzymes and serum anti-IgE antibody to cat allergen). A biomarker of effect is a measurable alteration of an endogenous factor that is shown to be linked with an impairment or disease, such as an increase in serum liver enzymes following exposure to carbon tetrachloride. A biomarker of susceptibility (or resistance) can be an indicator of an inherent or acquired property that 
alters the response of exposure to an endogenous agent. For example, individuals who lack glutathione transferases, enzymes that are involved in the detoxification of tobacco, are more susceptible to lung cancer. ${ }^{6}$

Biomarkers serve numerous functions. ${ }^{5}$ In the clinic setting, they can be used to identify whether a child's symptoms are due to an exposure or ingestion of an allergen or toxicant, such as hypersensitivity to an indoor allergen or pesticide poisoning. Biomarkers can be used to identify subgroups of children who are particular risk for exposures. ${ }^{5}$ They can be also used to provide an integrated measure of dose. Children's blood lead concentration, for example, is a result of cumulative exposure and ingestion of lead contaminated house dust, paint, soil, and water. ${ }^{13}$ Biomarkers can be used to conduct surveillance and identify trends in exposures. Biomonitoring has shown that the concentration of brominated diphenyl ethers (PBDE), a flame retardant, has increased in human breast milk. ${ }^{14}$ Finally, they can be used to test the efficacy of various prevention efforts. Numerous studies have tested the efficacy of parental smoking cessation education on children's exposure to environmental tobacco smoke using urine, serum or hair cotinine. ${ }^{15} 16$

\section{BIOLOGICAL MATRICES}

Biomarkers can be measured in a variety of tissues and body fluids, or matrices. Biomarkers are routinely measured in blood or serum, saliva, urine, hair, and breast milk. Lead has been measured in bone, using $x$ ray fluorescence, and in shed deciduous teeth. Numerous biomarkers of exposure, including cocaine, lead, pesticides, alcohol metabolites, and tobacco are being measured in meconium to quantify fetal exposure to various toxicants. ${ }^{17}$ Still, many of these biomarkers have not been validated for assessing exposure or their ability to predict disability or disease.

Establishing the optimal matrix is based on a variety of considerations. The absorption, distribution, metabolism, and excretion of an exogenous substance influences what tissues or body fluids should be used to measure biomarkers. ${ }^{5}$ For example, persistent chemicals are more readily measured in blood whereas non-persistent chemicals are often easier to measure in urine. Lipophilic substances are readily found in breast milk. The magnitude, frequency, and duration of exposure also influence what tissues should be used to measure biomarkers. Indeed, the variability of environmental chemicals in biological samples may require multiple sampling to characterise exposures. In contrast with blood, urine, and saliva, hair offers a longer term measure of exposure (approx. $1 \mathrm{~cm}$ of hair is equivalent to one month of exposure) for toxicants with a short half-life, such as tobacco or organophosphate insecticides. Hair has been used to quantify exposure to cotinine and mercury. ${ }^{18}{ }^{19}$ Meconium offers several potential benefits as a cumulative measure of fetal exposure; it can be collected non-invasively, provides a direct measure of what enters the fetal compartment, and can be used to quantify numerous toxicants. ${ }^{17}$ Finally, the burden to the patient or research subject is an important consideration; if an invasive procedure, such as amniocentesis, is necessary to collect a specimen, it will obviously not be useful for routine screening tests or large epidemiological studies. The ultimate validity of a biomarker is the extent to which it can predict disease occurrence. ${ }^{20}$

\section{BIOMARKERS IN PAEDIATRIC RESEARCH}

Technologic advances in biomarkers for infectious agents led to rapid progress in diagnosing and controlling infectious diseases. Similarly, biomarkers will ultimately revolutionise our understanding of environmental causes for many childhood conditions and diseases. The remainder of this review highlights some of the research findings that used biomarkers to improve our understanding and control of environmentally induced disease and disability in children.

FETAL AND CHILDHOOD EXPOSURE TO TOXICANTS Toxicants can cross the placenta and enter the fetus. In the past, the placenta was believed to act as a protective barrier against toxicants. But the global epidemic of phocomelia (seal-limb) due to the drug thalidomide was a dire warning that many drugs and toxicants cross the placenta. ${ }^{21}$ Indeed, the unborn child is the recipient of lead, carcinogens, tobacco, mercury, persistent pesticides, and other toxicants from their mother. ${ }^{47}$ In some cases, such as with mercury exposure, the dose that gets into the fetus is greater than the mother's dose. ${ }^{22}$ The fetus may also be more vulnerable to the effects of the toxicants, such as the neurotoxic effects of methyl mercury and the carcinogenic effects of tobacco smoke exposure. $^{18} 23$

\section{REPRODUCTIVE TOXICITY}

It has long been recognised that heavy exposures of environmental toxicants are associated with adverse reproductive outcomes. ${ }^{724}$ Using biomarkers, researchers are finding that low level exposures to prevalent environmental toxicants are linked with adverse reproductive outcomes.

Active smoking during pregnancy is a recognised risk factor for lower birth weight and prematurity. ${ }^{25}{ }^{26}$ But some women and their children are at greater risk from tobacco exposure. Wang and co-workers showed, for example, that among pregnant women who actively used tobacco, only children who were exposed to active tobacco use and who had GSTTl $_{\text {null }}$ (a biomarker of susceptibility) were at increased risk for lower birth weight and preterm birth. ${ }^{27}$ Indeed, previous estimates of risk were averaged out over both susceptible and resistant populations. Our ability to identify susceptible individuals allows us to more accurately quantify the risk of tobacco exposure and other toxicants for pregnant women and children (fig 2). Although a parent's report of environmental tobacco smoke (ETS) exposure has been widely used to show that tobacco exposure is hazardous, some studies indicate that cotinine is a better predictor of decrements in birth weight. ${ }^{28}$ Using urine cotinine, England and her co-workers found that the greatest decrements in birth weight occurred at lower levels of exposure, equivalent to $<5$ cigarettes per day. ${ }^{25}$ Finally, Jakkola and his co-workers found, using hair nicotine, that exposure to ETS was a risk factor for prematurity. Pregnant women with $>4 \mu \mathrm{g} / \mathrm{g}$ of nicotine in their hair were at a sixfold increased risk for having a premature delivery compared with women in the reference group. ${ }^{29}$

There is also evidence that lead and other toxicants are reproductive toxicants. Lead has been associated with spontaneous abortion at blood lead levels considerably below $40 \mu \mathrm{g} / \mathrm{dl}$, the level considered acceptable for an adult woman. Compared with pregnant women whose blood lead concentration was $<5 \mu \mathrm{g} / \mathrm{dl}$, women who had blood lead levels between $10 \mu \mathrm{g} / \mathrm{dl}$ and $14 \mu \mathrm{g} / \mathrm{dl}$ were at a fivefold increased risk for spontaneous abortion. ${ }^{30}$ In an innovative study using maternal serum collected over 30 years ago, Longnecker and his colleagues found that p'p-DDE (a metabolite of DDT) was associated with premature birth in a dose dependent fashion. ${ }^{31}$ None of these studies would have been feasible but for the development of biomarker technology.

\section{DEVELOPMENTAL TOXICITY Lead toxicity}

The developing brain is especially vulnerable to some environmental toxicants. Environmental neurotoxicants have been linked with learning disabilities and behavioural 

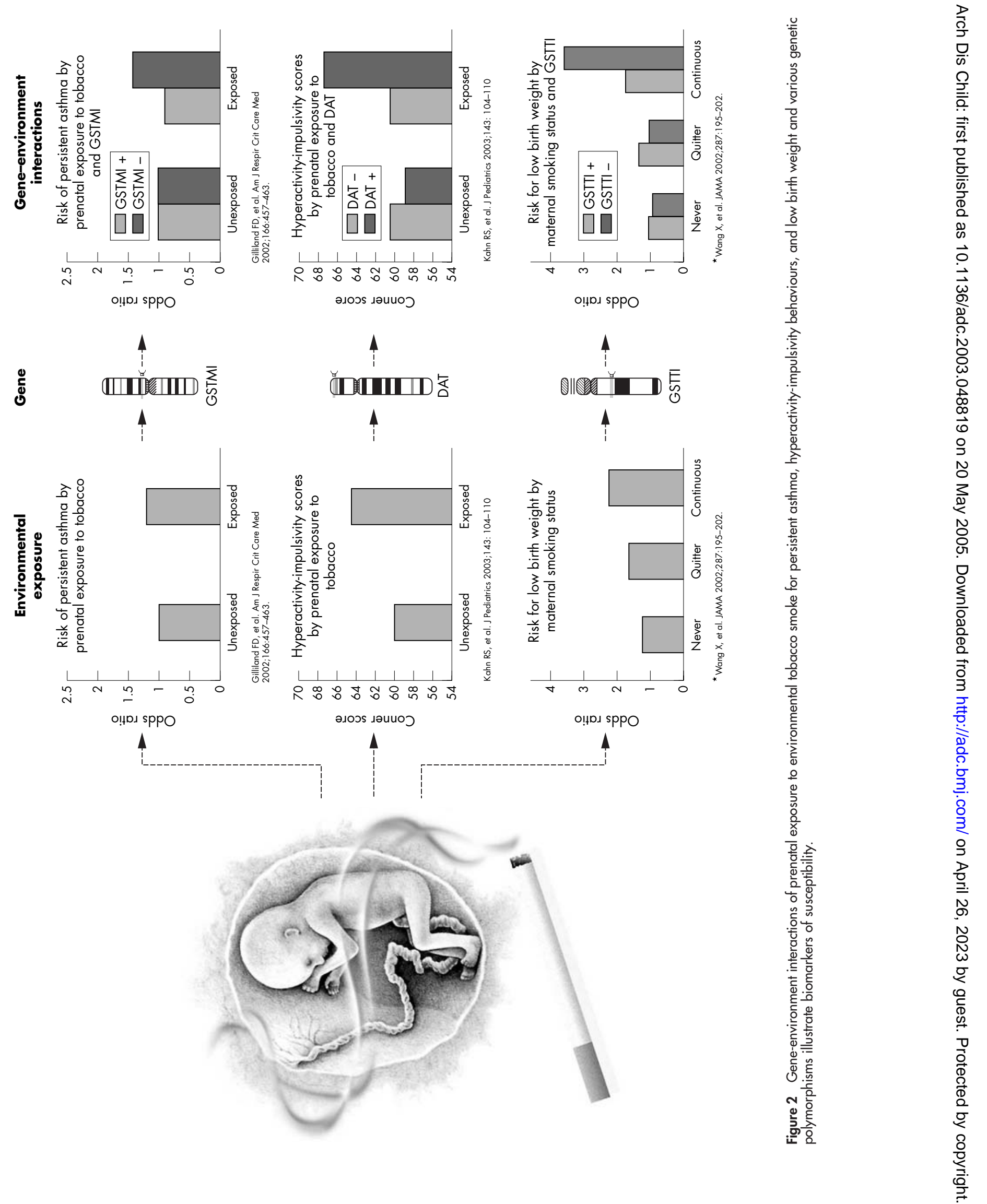
problems in children. In 1979, Needleman and his co-workers found that children with higher dentine (tooth) lead concentrations were more likely to be rated unfavourably by teachers on the dimensions of distractibility, organisational ability, dependence, impulsivity, daydreaming, and ability to follow directions. ${ }^{32}$ In a follow up study, they reported that children with higher dentine lead levels were found to have lower reading scores, lower class rank, and increased absenteeism in adolescence. Children in the higher lead group were 5.8 times more likely to have a reading disability and 7.4 times more likely to drop out of school than children in the lower group. ${ }^{33}$ In another prospective cohort study, childhood lead exposure was found to be a predictor of delinquent behaviours in adolescence. ${ }^{34}$

The use of biomarkers is increasingly important for examining low level exposures to environmental toxicants. There is emerging evidence that lead associated intellectual deficits occur at blood levels below $10 \mu \mathrm{g} / \mathrm{dl}$, the action level set by the World Health Organisation. ${ }^{35}$ Previous studies estimated that there was a 2.5-3 point IQ decrement linked with an increase in blood lead from $10 \mu \mathrm{g} / \mathrm{dl}$ to $20 \mu \mathrm{g} / \mathrm{dl} .^{36}$ More recently, using whole blood lead, an increase in children's lifetime mean blood lead level from $<1$ to $10 \mu \mathrm{g} /$ dl was associated with a 7.4 point IQ deficit. ${ }^{37}$ Moreover, the lead associated IQ decrement was greater for a given exposure at blood lead levels below $10 \mu \mathrm{g} / \mathrm{dl}$. Not only did these studies rely on blood lead as a biomarker, they required a high degree of analytical precision at low levels of exposure.

\section{Methyl mercury}

Methyl (organic) mercury is almost completely absorbed when ingested; it crosses the placenta easily and is stored by the fetus. ${ }^{182238}$ At high doses, fetal exposure can result in widespread developmental damage, such as mental retardation, spastic paralysis, and death. ${ }^{18}$ At lower doses, exposure has been linked with deficits in neuromotor performance, cognition, memory, and language in some, but not other studies. ${ }^{39}{ }^{40}$ One reason proposed for these differences is that some studies used hair as the biological matrix whereas others used cord blood. ${ }^{40}$

\section{Polychlorinated biphenyls (PCBs)}

Extremely high PCB exposure during fetal development causes low birth weight, dark pigmentation of the skin, early eruption of teeth, acneform rash, and death. ${ }^{7}$ Low level fetal exposure to PCBs has been associated with weak reflexes, poorer visual recognition memory, delayed motor development, poor memory functioning, and diminished information processing abilities. ${ }^{71}$ Using cord blood or maternal breast milk, investigators have found that prenatal PCB exposure was associated with deficits in intelligence, memory, and attention. ${ }^{41}{ }^{42}$

\section{Tobacco exposure}

Prenatal tobacco exposure is a risk factor for behavioural problems, such as attention deficit hyperactivity disorder (ADHD), and lowered intellectual ability. ${ }^{43} 44$ In one study, Wakschlag found that mothers who smoked during pregnancy were four times more likely to have a child with a conduct disorder. ${ }^{43}$ Kahn and his colleagues found that only children who were exposed in utero to tobacco and who had dopamine transporter (DAT +/+) were at increased risk for features consistent with ADHD and oppositional behaviours. ${ }^{45}$ At exceedingly low levels of exposure to ETS-levels that were, until fairly recently, below the level of detectionYolton and co-workers found that postnatal exposure to ETS was associated with lower reading scores. ${ }^{46}$ Moreover, the decrements in reading scores were, for a given increment in exposure, greater at lower serum cotinine levels.

\section{Ethanol}

Ethanol, the alcohol in alcoholic beverages, readily crosses the placenta and causes harm to the fetus. ${ }^{47}{ }^{48}$ At high doses, alcohol consumption causes fetal alcohol syndrome. ${ }^{49}$ However, the effects of low doses are increasingly being recognised, with $1 \%$ of all newborns estimated to exhibit some prenatal alcohol damage. ${ }^{50}$ But it is difficult to quantify alcohol intake during pregnancy. In the future, biomarkers of prenatal ethanol exposure, fatty acid ethyl esters in meconium and hair, may improve the quantification of maternal drinking and any effects at lower levels. ${ }^{51-54}$

\section{PULMONARY TOXICITY \\ Asthma}

Asthma is one of the most prevalent and disabling disease of children. ${ }^{55}$ The prevalence of asthma has increased considerably during the last two decades, for reasons that are largely unexplained. ${ }^{56}$ Using serum cotinine, over 150000 cases of asthma in preschool children were attributed to ETS exposure. $^{57}$ The increased prevalence of asthma cannot, however, be explained by an increase in ETS exposure. Indeed, studies from both England and the USA show dramatic reductions in children's cotinine levels. ${ }^{58} 59$ There is also substantial evidence, using skin test hypersensitivity (a biomarker of effect) and serum cotinine that links exposures to indoor pollutants with childhood asthma. ${ }^{60}$ It was estimated that over $40 \%$ of doctor diagnosed asthma can be attributed to housing exposures, including indoor allergens and ETS. ${ }^{60}$ But not all children are equally susceptible to specific respiratory toxicants. Gilliland and co-workers have shown that only children who were exposed in utero to tobacco and who had GSTMI $\mathrm{I}_{\text {null }}$ were at increased risk for persistent asthma and wheezing ${ }^{61}$ (fig 2).

\section{RESPIRATORY INFECTIONS}

Exposure to environmental tobacco smoke is a well established risk factor for respiratory infections. ${ }^{62}$ Still, most studies relied on parent reported exposure to ETS. In a striking illustration of the potential value of biomarkers, Nuesslein and co-workers found that the amount of cotinine in meconium, a cumulative biomarker of in utero exposure during the second and third trimester of pregnancy, was a predictor of upper $(\mathrm{OR}=12.5)$ and lower respiratory tract infections $(\mathrm{OR}=4.9)$ during infancy. ${ }^{63}$ In contrast, neither the maternal report of active smoking or passive exposure to tobacco smoke was risk factors for respiratory infections in infancy in adjusted logistic regression analyses that included meconium cotinine.

\section{CARCINOGENICITY}

There is some evidence that children are susceptible to the carcinogenic effects from environmental tobacco smoke. Young children have significantly higher concentrations of serum cotinine and 1-hydroxypyrene (a biomarker for PAH exposures from industrial emissions, automobile exhaust, and tobacco smoke) than non-smoking adults. ${ }^{10}$ The higher levels of biomarkers in children could be due to differences in metabolism or increased respiratory rates. ${ }^{64}$ But young children may also ingest nicotine contaminated house dust. ${ }^{65}$

Although the fetus is exposed to significantly lower levels of PAH, the level of DNA damage in the fetus (measured using DNA adducts in cord blood) is 10-fold higher than expected. ${ }^{23}$ DNA adducts in WBCs have been shown, in a prospective study of adults, to be associated with an increased risk for developing lung cancer. ${ }^{66}$ Still, the evidence linking ETS exposure in childhood as a risk factor for lung cancer in adulthood is mixed. ${ }^{67}$ The failure to show an effect may be due to resiliency of children (that is, diminished risk 
from remote exposure) or failure to examine susceptible children; several studies in adults have shown that individuals who are unable to detoxify or rapidly metabolise tobacco products are at an increased risk for lung cancer. ${ }^{666}$

\section{BIOMARKERS IN CLINICAL PRACTICE}

It is often difficult for a parent or a paediatrician to accurately assess their child's exposure to toxicants; indeed, children's exposures to pollutants and other environmental influences are often insidious. For some influences, such as tobacco exposure, parents often have a good idea about their child's exposure. For other toxicants, such as lead, we are heavily dependent on using biomarkers to assess a child's exposure, diagnose a condition, and ascertain the need for therapy.

Biomarkers are not yet established for many toxicants. ${ }^{12} 17$ Even with established biomarkers, it is not always clear which biological matrix should be used. For children, urine and serum cotinine are the gold standard for measuring ETS exposure, but hair cotinine or hair nicotine may offer a longer term measure of exposure that is more predictive of certain outcomes. Whole blood is the accepted matrix for measuring environmental lead exposure in children. Fetal exposure has typically been measured using maternal whole blood or umbilical cord blood. But these methods may not be the best indicator of the dose that reaches the fetus or of cumulative fetal exposure. Some scientists are using maternal plasma lead and bone lead as a measure of fetal exposure. ${ }^{68}$ Meconium and maternal hair have been used as biological matrices for alcohol, cocaine, and ETS, and they hold promise as non-invasive biomarkers to test for exposure to numerous toxicants during fetal development. ${ }^{1722}{ }^{69}$ Still, it is unknown whether conventional biological matrices, hair, or meconium are more predictive of the adverse effects from fetal exposures to specific toxicants.

\section{SCREENING FOR LEAD TOXICITY}

Numerous challenges remain after a biomarker is established for an environmental toxicant, such as lead. In the United States, the vast majority of paediatricians recognise lead as a toxicant, but less than half screen children's blood for excessive exposure to lead..$^{70}$ There are numerous reasons for paediatricians' paradoxical behaviour. First, few children are identified as having an "elevated" blood lead level in some clinics or communities. ${ }^{71}$ Even when there is an established biomarker for a confirmed toxicant, there is a need to conduct targeted screening and confirm that using the test identifies children who are at risk. Second, venipuncture is an inexpensive and routine test, but many paediatric clinics do not have a phlebotomist. Finally, uncertainties about the safety and efficacy of lead hazard controls or educational interventions may inhibit paediatricians from routinely screening children for lead toxicity. ${ }^{72}$

\section{REDUCING CHILDREN'S EXPOSURE TO ETS}

Biomarkers have been essential to test efforts to reduce children's ETS exposure. Physicians routinely ask parents to stop smoking. Unfortunately, smoking cessation was no more successful if parents were provided with a physician's feedback of the cotinine levels in their child's urine or given specific information regarding the risks to their child's health. ${ }^{15}{ }^{16}$ In the absence of cessation, we often counsel parents to smoke outdoors. Until recently, however, it was unknown if this led to a significant reduction in children's exposure to ETS. Indeed, smoking outdoors only appears to reduce children's exposure to tobacco smoke, as measured using cotinine, if the restrictions are absolute and tobacco use is banned entirely from the home. ${ }^{73}{ }^{74}$ It is therefore not clear that screening children for an elevated cotinine level would be useful for the management of children with asthma or other tobacco related diseases.

\section{CONCLUSIONS}

Over the past century, increasing evidence has emerged linking chronic, low level exposure to environmental influences and industrial toxicants with many of the "new morbidities of childhood", including intellectual deficits, lowered birth weight, prematurity, and behavioural problems, such as ADHD, delinquency, and asthma. Ultimately, the aetiology and prevention of human disease can only be established in the context of both genetic susceptibility and environmental factors. Indeed, the contribution of genetic factors may only become evident once environmental exposures are taken into account. Similarly, the risks associated with exposure to an environmental agent may only be evident when susceptible children are examined. Paediatricians are increasingly asked by parents for advice about how to prevent, control, or treat an array of conditions linked with environmental exposures. If paediatricians aim to maintain their orientation to prevention, they will need to play a larger role in identifying and controlling children's exposure to environmental agents. In the future, biomarkers will offer increasing opportunities to investigate, prevent, diagnose, and treat environmentally induced diseases and disabilities in children.

\section{ACKNOWLEDGEMENTS}

We acknowledge Robert Kahn, MD, for many hours of enjoyable discussion about how to present results of gene-environment interaction studies.

\section{Authors' affiliations}

B P Lanphear, Cincinnati Children's Environmental Health Center, Cincinnati Children's Hospital Medical Center, Departments of Pediatrics and of Environmental Health, University of Cincinnati, Cincinnati, Ohio, USA

C F Bearer, Departments of Pediatrics and of Neurosciences, Mary Ann Swetland Center for Environmental Health, Case Western Reserve University School of Medicine, Rainbow Babies \& Children's Hospital, Cleveland, Ohio, USA

Funding: funded in part by NIEHS and US EPA (PO1-ES11261)

Competing interests: none declared

\section{REFERENCES}

1 Willett WC. Balancing life-style and genomics research for disease prevention. Science 2002;296:695-8.

2 Lichtenstein P, Holm NV, Verkasalo PK, et al. Environmental and heritable factors in the causation of cancer-analyses of cohorts of twins from Sweden, Denmark, and Finland. N Engl J Med 2000;343:78-85.

3 Valent $F$, Little $D$, Bertonelli $R$, et al. Burden of disease attributable to selected environmental factors and injury among children and adolescents in Europe. Lancet 2004;363:2032-9.

4 Landrigan PJ, Carlson JE, Bearer CF, et al. Children's health and the environment: a new agenda for prevention research. Environ Health Perspect 1998;106(suppl 3):787-94.

5 Sexton K, Needham LL, Pirkle JL. Human biomonitoring of environmental chemicals. American Scientist 2004;92:38-45.

6 Perera FP. Environment and cancer: who are susceptible? Science 1997;278: 1068-73.

7 Rogan WJ. Environmental poisoning of children: lessons from the past. Environ Health Perspect 1995; 103(suppl 6):19-23.

8 Rose, G. The strategy of preventive medicine. Oxford: Oxford University Press, 1992.

9 Links JM, Kensler TW, Groopman JD. Biomarkers and mechanistic approaches in environmental epidemiology. Annu Rev Public Health 1995; 16:83-103.

10 Centers for Disease Control. Second national report on human exposure to environmental chemicals, US Public Health Service, Department of Health and Human Services, 2003.

11 Bearer CF. Biomarkers in pediatric environmental health: a cross-cutting issue. Environ Health Perspect 1998;106(suppl 3):813-16.

12 Groopman JD, Kensler KW. The light at the end of the tunnel for chemicalspecific biomarkers: daylight or headlight? Carcinogenesis 1999;20:1-11.

13 Lanphear BP, Hornung $R$, Ho $M$, et al. Environmental lead exposure during early childhood. J Pediatr 2002;140:40-7. 
14 Noren K, Meironyte D. Contaminants in Swedish human milk: decreasing levels of organochlorines and increasing levels of organobromine compounds. Organohalogen Compounds 1998;38:1-4.

15 Wakefield M, Banham D, McCaul K, et al. Effect of feedback regarding urinary cotinine and brief tailored advice on home smoking restrictions among low-income parents of children with asthma: a controlled trial. Prev Med 2002;34:58-65.

16 Conway TL, Woodruff SI, Edwards CC, et al. Intervention to reduce environmental tobacco smoke exposure in Latino children: null effects on hair biomarkers and parent reports. Tob Control 2004;13:90-2.

17 Bearer CF. Meconium as a biological marker of prenatal exposure. Ambulatory Pediatrics 2003;3:40-3.

18 Clarkson TW, Magos L, Meyers GJ. The toxicology of mercury-current exposures and clinical manifestations. N Engl J Med 2003;349:1731-7.

19 Eliopoulos C, Klein J, Koren G. Validation of self-reported smoking by analysis of hair for nicotine and cotinine. Ther Drug Monit 1996;18:532-6.

20 Hulka BS, Margolin BH. Methodologic issues in epidemiologic studies using biologic markers. Am J Epidemiol 1992:135:200-9.

21 Wilson JG. The evolution of teratological testing. Teratology 1979;20:205-11.

22 Ramirez GB, Cruz MC, Pagulayan O, et al. The Tagum study I: analysis and clinical correlates of mercury in maternal and cord blood, breast milk, meconium, and infants' hair. Pediatrics 2000;106:774-81.

23 Whyatt RM, Jedrychowski W, Hemminki K, et al. Biomarkers of polycyclic aromatic hydrocarbon-DNA damage and cigarette smoke exposures in paired maternal and newborn blood samples as a measure of differential susceptibility. Cancer Epidemiol Biomark Prevent 2001;10:581-8.

24 Institute of Medicine. The role of environmental hazards in premature birth Washington, DC: National Academies Press, 2003.

25 England LJ, Kendrick JS, Wilson HG, et al. Effects of smoking reduction during pregnancy on the birth weight of term infants. Am J Epidemiol 2001:154:694-701.

26 Hofius W, de Jongste JC, Merkus PJFM. Adverse health effects of prenatal and postnatal tobacco smoke exposure on children. Arch Dis Child 2003;88:1086-90.

27 Wang X, Zuckerman B, Pearson C, et al. Maternal cigarette smoking, metabolic gene polymorphism, and infant birth weight. JAMA 2002;287:195-202.

28 Klebanoff MA, Levine RJ, Clemens JD, et al. Serum cotinine concentration and self-reported smoking during pregnancy. Am J Epidemiol 1998;148:259-62.

29 Jaakkola JJK, Jaakkola N, Zahlsen K. Fetal growth and length of gestation in relation to prenatal exposure to environmental tobacco smoke assessed by hair nicotine concentration. Environ Health Perspect 2001;109:557-61

30 Borja-Aburto VH, Hertz-Picciotto I, Rojas Lopez M, et al. Blood lead levels measured prospectively and risk of spontaneous abortion. Am J Epidemiol 1999:150:590-7.

31 Longnecker MP, Klebanoff MA, Zhou $\mathrm{H}$, et al. Association between materna serum concentration of the DDT metabolite DDE and preterm and small-forgestational-age babies at birth. Lancet 2001;358:110-14.

32 Needleman HL, Gunnoe C, Leviton A, et al. Deficits in psychologic and classroom performance of children with elevated dentine lead levels. N Engl J Med 1979:300:689-95.

33 Needleman $\mathrm{HL}$, Schell A, Bellinger D, et al. The long-term effects of exposure to low doses of lead in childhood. An 11-year follow-up report. N Engl J Med 1990;322:83-8.

34 Dietrich K, Ris $M$, Succop $P$, et al. Early exposure to lead and juvenile delinquency. Neurotox Teratol $2001 ; 23: 511-18$.

35 World Health Organisation, International Programme on Chemical Safety Environmental health criteria 165-inorganic lead. Geneva: WHO, 1995.

36 Schwartz J. Low-level lead exposure and children's IQ: search for a threshold. Environ Res 1994:65:42-55.

37 Canfield RL, Henderson CR, Cory-Slechta DA, et al. Intellectual impairment in children with blood lead concentrations below 10 micrograms per deciliter. N Engl J Med 2003;348:1517-26.

38 Mahaffey KR, Clickner RP, Bodurow CC. Blood organic mercury and dietary mercury intake: National Health and Nutrition Examination Survey, 1999 and 2000. Environ Health Perspect 2004; 1 12:562-70.

39 Grandjean P, Weihe P, White RF, et al. Cognitive deficit in 7-year-old children with prenatal exposure to methylmercury. Neurotoxicol Terato 1997:19:417-28.

40 Myers GJ, Davidson PW, Cox C, et al. Prenatal methylmercury exposure from ocean fish consumption in the Seychelles child development study. Lancet 2003;361:1686-92.

41 Jacobson JL, Jacobson SW. Intellectual impairment in children exposed to polychlorinated biphenyls in utero. N Engl J Med 1996;335:783-9.

42 Schantz SL, Widholm JJ, Rice DC. Effects of PCB exposure on neuropsychological function in children. Environ Health Perspect 2003;1 11:357-76.

43 Wakschlag LS, Pickett KE, Cook E Jr, et al. Maternal smoking during pregnancy and severe antisocial behavior in offspring: a review. Am J Public Health 2002;92:966-74.
44 Weitzman M, Byrd RS, Aligne CA, et al. The effects of tobacco exposure on children's behavioral and cognitive functioning: implications for clinical and public health policy and future research. Neurotoxicol Teratol 2002;24:397-406

45 Kahn RS, Khoury J, Nichols WC, et al. Role of dopamine transporter genotype and maternal prenatal smoking in childhood hyperactive-impulsive, inattentive, and oppositional behaviors. J Pediatr 2003;143:104-10

46 Yolton $\mathrm{K}$, Dietrich $\mathrm{K}$, Auinger $\mathrm{P}$, et al. Exposure to environmental tobacco smoke and cognitive abilities among US children and adolescents. Environ Health Perspect 2005;113:98-103.

47 Brien JF, Clarke DW, Richardson B, et al. Disposition of ethanol in maternal blood, fetal blood, and amniotic fluid of third-trimester pregnant ewes. Am J Obstet Gynecol 1985;152:583-90.

48 Abel EL, Sokol RJ. A revised conservative estimate of the incidence of FAS and its economic impact. Alcohol Clin Exp Res 1991;15:514-24.

49 Jones KL, Smith DW. Recognition of the fetal alcohol syndrome in early infancy. Lancet 1973;2:999-1001.

50 Sampson PD, Streissguth AP, Bookstein FL, et al. Incidence of fetal alcohol syndrome and prevalence of alcohol-related neurodevelopmental disorder. Teratology 1997;56:317-26.

51 Bearer CF, Lee S, Salvator AE, et al. Ethyl linoleate in meconium: a biomarker for prenatal ethanol exposure. Alcohol Clin Exp Res 1999;23:487-93.

52 Klein J, Karaskov T, Koren G. Fatty acid ethyl esters: a novel biologic marker for heavy in utero ethanol exposure: a case report. Ther Drug Monit 1999;21:644-6.

53 Chan D, Caprara D, Blanchette $\mathrm{P}$, et al. Recent developments in meconium and hair testing methods for the confirmation of gestational exposures to alcohol and tobacco smoke. Clin Biochem 2004;37:429-38.

54 Bearer CF, Jacobson JL, Jacobson SW, et al. Validation of a new biomarker of fetal exposure to alcohol. J Pediatr 2003;143:463-9.

55 Newacheck PW, Halfon N. Prevalence, impact, and trends in childhood disability due to asthma. Arch Pediatr Adolesc Med 2000; 154:287-93.

56 Akinbami LJ, Schoendorf KC. Trends in childhood asthma: prevalence, health care utilization, and mortality. Pediatrics 2002;1 10:315-22.

57 Gergen P, Fowler JA, Maurer KR, et al. The burden of environmental tobacco smoke exposure on the respiratory health of children. Pediatrics 1998;101:e8.

58 Pirkle JL, Flegal KM, Bernert JT, et al. Exposure of the US population to environmental tobacco smoke. JAMA 1996;275:1233-40.

59 Jarvis MJ, Goddard E, Higgins, et al. Children's exposure to passive smoking in England since the 1980s: cotinine evidence from population surveys. BMJ 2000;321:343-5

60 Lanphear BP, Kahn RS, Berger O, et al. Contribution of residential exposures to asthma in US children and adolescents. Pediatrics 2001;107:e98.

61 Gilliand FD, Li Y, Dueau L, et al. Effects of glutathione S-transferase MI maternal smoking during pregnancy, and environmental tobacco smoke on asthma and wheezing in children. Am J Respir Crit Care Med 2002; 166:457-63.

62 Difranza JR, Lew RA. Morbidity and mortality associated with use of tobacco products by other people. Pediatrics 1996;97:560-8.

63 Nuesslein TG, Beckers D, Rieger CH. Cotinine in meconium indicates risk for early respiratory tract infections. Hum Exp Toxicol 1999; 18:283-90.

64 Bearer CF. How are children different from adults? Environ Health Perspect 1995; 103(suppl 6):7-12.

65 Matt GE, Quintana PJ, Hovell MF, et al. Household contaminated by environmental tobacco smoke: sources of infant exposures. Tob Control 2004; 13:29-37.

66 Perera FP, Mooney LA, Stampfer M, et al. Associations between carcinogenDNA damage, glutathione S-transferase genotypes, and risk of lung cancer in the prospective Physicians' Health Cohort Study. Carcinogenesis 2002;23:1641-6.

67 Boffetta P, Tredaniel J, Greco A. Risk of childhood cancer and adult lung cancer after childhood exposure to passive smoke: a meta-analysis. Environ Health Perspect 2000;108:73-82.

68 Hu H, Rabinowitz M, Smith D. Bone lead as a biological marker in epidemiologic studies of chronic toxicity: conceptual paradigms. Environ Health Perspect 1998;106:1-8.

69 Ostrea EM Jr, Knapp DK, Tannenbaum L, et al. Estimates of illicit drug use during pregnancy by maternal interview, hair analysis, and meconium analysis. J Pediatr 2001;138:344-8.

70 Campbell JR, Schaffer SJ, Szilagyi PG, et al. Blood lead screening practices among US pediatricians. Pediatrics 1996;98:372-7.

71 Harvey B. Should blood lead screening recommendations be revised? Pediatrics 1994;93:201-4.

72 Lanphear BP, Dietrich KN, Berger O. Prevention of lead toxicity in US children. Ambulatory Pediatrics 2003;3:27-36.

73 Wakefield M, Baham D, Ruffin MJ, et al. Restrictions on smoking at home and urinary cotinine levels among children with asthma. Am J Prev Med 2000; 19:188-92.

74 Johansson A, Hermanson G, Ludvigsson J. How should parents protect their children from environmental tobacco smoke? Pediatrics 2004;113:e291. 